\begin{abstract}
APERTURA DE LOS ESPACIOS DE PARTICIPACIÓN DE LA SOCIEDAD EN EL MANEJO SUSTENTABLE DEL RECURSO HÍDRICO PARA ASEGURAR EL BIENESTAR SOCIAL DE LOS HABITANTES EN LAS COMUNIDADES. EXPERIENCIA DE CASO: "LA CUENCA DEL RÍO SONORA"
\end{abstract}

\author{
Adria Velia GONZÁLEZ BELTRONES ${ }^{1}$ \\ Guadalupe Aleida VALENZUELA MIRANDA ${ }^{2}$ \\ Jesús Miguel MAYA RODRÍGUEZ ${ }^{3}$
}

SUMARIO: I. Resumen. II. Introducción. III. Objeto. IV. Justificación. V. Experiencia de caso: Parte Primera. A) Actores locales. B) Actores Federales. VI Antecedentes y precisiones de la trama del tema que nos ocupa. VII. Parte Segunda La reforma legislativa de 2004 en México. VIII. ¿Qué requieren hacer los gobiernos para que las medidas sean efectivas?. IX. Referencias Bibliográficas.

\title{
I. Resumen
}

En este trabajo se pretende destacar la importancia de la apertura de los espacios de participación de la sociedad en el manejo sustentable del recurso hídrico, su consolidación a través de la expedición urgente de los reglamentos y manuales que señala la propia Ley de Aguas Nacionales LAN para que los ciudadanos y las organizaciones puedan potenciar su actuación de manera eficiente y corresponsable y al mismo tiempo poder exigir de las autoridades transparencia de la eficacia en su manejo.

\footnotetext{
${ }^{1}$ Doctora en Ciencias Políticas y Sociales por la UNAM. Investigador de Tiempo Completo, Categoría Titular "C". Pertenece al Grupo de Investigación en Estudios en Ciencias Sociales CIECS. Departamento de Derecho Universidad de Sonora México. http://www.ciiacs.uson.mx E-mail: adriag@sociales.uson.mx.

${ }^{2}$ Doctora en Educación por la UNED. Investigador de Tiempo Completo, Categoría Titular "A". Pertenece al Grupo de Investigación en Estudios en Ciencias Sociales CIECS. Departamento de Derecho Universidad de Sonora México. http://www.ciiacs.uson.mx Email: avalenzuela@sociales.uson.mx.

${ }^{3}$ Lic. Derecho por la UNISÓN. Estudiante de la Maestría en Innovación Educativa. Investigador Asociado Adjunto. aspire3000@hotmail.com.
} 
This paper is intended to highlight the importance of the opening of spaces for the participation of society in sustainable water resources management, its consolidation through the issuance of urgent regulations and manuals referred by the law of national waters LAN so that citizens and organizations may enhance its performance efficiently and jointly responsible and at the same demand the authorities efficiency and transparency in its management.

\section{Introducción}

El agua, como recurso natural integral de los ecosistemas en su dinámica natural en las zonas por donde fluye, si bien no reconoce fronteras geopolíticas si juega un papel importante en el desarrollo social y económico de las naciones definiendo una serie de interacciones de tal complejidad que demandan conocimiento para su manejo efectivo. El significado del agua, ciertamente ha sufrido cambios dramáticos en nuestro país durante las últimas seis décadas. "Mientras que en 1947 se sostenía que era obligación del Estado encabezar el aprovechamiento científico del agua para hacer de ella un bastión del progreso nacional, en la década de los noventa se decía, en cambio, que el Estado debía atender una extrema crisis hidráulica marcada por la escasez, la contaminación y el derroche. Mientras que en 1950 reinaba el optimismo de los ingenieros-políticos las grandes posibilidades que según ellos abría al país la expansión de los usos del agua, a principios del presente Siglo XXI prevalece un pesimismo que llevó a declarar el agua como asunto de seguridad nacional" "Ciertamente, este cambio de significado se relaciona con el crecimiento de la población ya que entre 1950 y 2000 la población mundial se triplicó, pasando de 2500 a 6000 millones. En México se aumentó de 25 a 100 millones de personas." ${ }^{5}$

\section{Objeto}

\footnotetext{
${ }^{4}$ Aboites Aguilar,Luis, El agua de la nación. Una historia política de México (1888-1946) pp. 89 México Ed. Centro de Investigaciones y Estudios Superiores de Antropología Social, 1998.

${ }^{5}$ González Beltrones Adria Agua: Asunto de seguridad nacional e importancia de la participación ciudadana en su gestión Cuestiones Jurídico Administrativas Locales, en Materia de Aguas del Siglo XX pp. 160, González Beltrones Adria Velia y Aragón Salcido María Inés, Ed. UNISON, 2010.
} 
En este trabajo se pretende significar la importancia de la efectiva participación de la sociedad en el manejo sustentable del recurso hídrico para asegurar el bienestar social de los habitantes en las comunidades.

\section{Justificación}

A finales del siglo pasado, una de las más importantes transformaciones de nuestras sociedades se dio en la revalorización de lo local. Paradójicamente, en un mundo en plena globalización, unificación mundial y desdibuja miento de fronteras, surge también un movimiento impulsor de las entidades locales y regionales, como un mecanismo social protector de su entorno próximo, de su ámbito del día a día, de su necesidad de pertenencia y de subsistencia.

La noticia de los problemas globales de trascendencia mundial pero traducida en preocupaciones y soluciones locales, parece ser una búsqueda de reposicionamiento social ante un mundo demasiado complejo en su desarrollo, en sus desigualdades y contradicciones. Es en éste escenario en donde lo nacional recupera su esencia simbólica como suma de localidades. El ejemplo más claro lo encontramos en la unificación europea a finales del siglo pasado, pero en América Latina aunque débiles, también se observan procesos de reacomodo, de celebración de alianzas, de mayor participación de la sociedad civil, de mayor fortalecimiento de las regiones, de resurgimiento de lo local como el eje de la acción social.

El signo distintivo en la región latinoamericana ha sido impulsar los procesos descentralizadores para fortalecer a los gobiernos locales, y a pesar de no haberse logrado en su totalidad el establecimiento de una nueva dinámica de relación entre el centro y las localidades, ha correspondido a éstas últimas y a la sociedad civil, paulatinamente influir, desde su ámbito local generación de propuestas y soluciones a la diversa problemática que afrontan, destacando la relacionada con el agua.

Es en éste escenario en el que debemos reconocer que el diseño de políticas e instrumentos que permitan el uso racional y eficiente; calidad y explotación adecuadas 
del recurso hídrico, no debe provenir exclusivamente del centro, del nivel federal, sino con la participación de los gobiernos locales y de la sociedad civil.

En la nueva concepción del papel del Estado, corresponde al centro convertirse en un articulador de esfuerzos locales (estado - municipio) y facilitador de los recursos y acciones de fortalecimiento. $Y$ al nivel local conducir la reforma necesaria, su orientación, modalidades y tiempos, si se quiere, que prevalezca el Estado - Nación. Pues si bien es cierto que históricamente en México como en otros países fue necesario centralizar para consolidar al Estado - Nación, en nuestros días, por la misma razón se requiere impulsar una redistribución no solamente de los recursos sino también del poder político.

La anterior reflexión, obedece a una tendencia que aunque no del todo generalizada en nuestro país ha inducido no solo en materia de manejo del agua, movimientos de reclamos del fortalecimiento de los gobiernos locales, y aun cuando algunas regiones se muestran más estables que otras, en el espacio municipal surgen experiencias demostrativas a su vez de esta nueva dinámica. Es decir, se presentan casos en los que el municipio adquiere cada vez mayor movilidad tanto en la participación ciudadana como en la obligada respuesta de los gobiernos locales y federal a sus demandas, traducida en la necesidad de nuevas formas de relación, nuevos mecanismos de gestión, nuevas estructuras de administración, nuevas soluciones y nuevas estrategias para preservar la gobernabilidad.

Sin embargo y para desesperación de muchos la falta de voluntad política en ocasiones o por cuestiones económicas en otras, las propuestas de soluciones a las problemáticas si bien locales de repercusión nacional natural, no logran su implementación. Por ejemplo,

\section{Experiencia de caso: Parte Primera A) Actores locales}

Hace cinco años el 26 de abril en la "sección metro" del periódico local sonorense "Expreso", apareció en toda una página, el siguiente encabezado: PRETENDE EL 
ESTADO LA ADMINISTRACIÓN DEL RECURSO HÍDRICO. Y al interior de la nota se escribió "Está lesionada la gobernabilidad hidráulica en la cuenca del Río Sonora" afirmó José Luis Jardines Moreno, en aquél entonces director general de la Comisión Estatal del Agua (CEA), quien continuó apuntando "entendiendo la gobernabilidad como el conjunto de reglas de una sociedad para poder vivir en armonía, en forma productiva, libre trascendente y creativa". Desde su punto de vista, tanto gobernantes como gobernados somos culpables del deficiente aprovechamiento del agua que escurre a través de la cuenca del Río Sonora, situación que el ex gobernador de Sonora Eduardo Bours Castelo comenzó a analizar desde su campaña como candidato al Gobierno del Estado esto es, en el año 2003. El resultado del precitado análisis motivó al ex mandatario estatal a realizar una petición inédita en la historia de México:

"Que se permita al Gobierno del Estado administrar el recurso hidráulico de la cuenca del Río Sonora".

De recibir una respuesta favorable por parte de las autoridades federales, Sonora se convertiría en la primera entidad federativa en participar en la administración del vital líquido. Por citar una tarea aparentemente sencilla cómo lo es el medir su uso o consumo, pero que en los hechos no lo es. Por lo que es válido señalar que en la cuenca del Río Sonora no se realiza satisfactoriamente la determinación de parámetros como temperatura, volumen de precipitación, evaporación, que deben de sumarse al registro consecutivo del número de días con lluvia apreciable, tormenta, granizo y niebla, entre otros. Situación que al decir del ex director de la Comisión Estatal del Agua "deriva de la ausencia de Estaciones Climatológicas e Hidrométricas y la mala administración de las existentes," acorde a los resultados de una revisión hecha por la CEA en la cuenca del Río Sonora, la cual puso al descubierto que, tanto estaciones climatológicas como hidrométricas, que son las que proporcionan los elementos básicos para una correcta planeación del uso del recurso hidráulico, no están funcionando en sus niveles óptimos. "No todas las mediciones que se deben hacer en esas estaciones se están haciendo", expresó, añadiendo que a "la problemática se suma que sólo cerca del $50 \%$ de los pozos de riego cuentan con medidor $y$, de éstos no funcionan más del 
$30 \% . . .$. Se desconocen las causas (de este rezago). Lo cierto es que no se cuenta con una medición de la extracción de agua"... Por su parte,

\section{B) Actores Federales}

Roberto Fernando Salmón Castelo, gerente central y regional de la Comisión Nacional del Agua (CNA) en el año 2006, reconoció también estas deficiencias, atribuyéndolas a "que la dependencia ha encabezado una tarea titánica que le permita regularizar los aprovechamientos de agua a nivel nacional" apuntando de igual manera el funcionario federal que "la falta de recursos humanos que permitan el permanente monitoreo en las estaciones de medición, así como la implementación de una Ley de Aguas Nacionales que data del año de 1992, la cual fue reformada en el 2003, son algunos de los factores que, sumados a la insuficiencia presupuestaria, han impedido el cumplimiento estricto de una ley vanguardista que propone un reordenamiento en la administración del recurso hídrico."

El precitado funcionario federal además informó, "que en la región sólo existen seis Estaciones Climatológicas y que en cada una existe personal capacitado en la realización de los monitoreos; pero también reconoció que a lo largo de la cuenca del Río Sonora se dificulta la realización de estos monitoreos, simplemente porque no se cuenta con estas estaciones de medición." Más de un lustro ha transcurrido desde que se emitieran tan hirientes declaraciones y como en el intermedio de los extremos de toda historia a continuación listamos brevemente lo que aconteció previa mención de los

\section{Antecedentes y precisiones de la trama del tema que nos ocupa:}

- Escasez de agua dulce es uno de los siete problemas ambientales fundamentales según el Informe del Programa de Naciones Unidas para el Medio Ambiente (PNUMA) (2007) 
- Mientras se alcanza el límite de extraer agua dulce de la superficie terrestre, el consumo no deja de aumentar.

Desafíos: Lograr el equilibrio hidrológico que asegure el abasto de agua a la población. Equilibrio que solo se alcanzará

- Armonizando la disponibilidad natural con las extracciones del recurso mediante el uso eficiente del agua.

- Contrarrestando el efecto que el cambio climático tendrá sobre el ciclo hidrológico y la disponibilidad de agua dulce.

Al respecto: El Informe de la UNESCO "El agua en un mundo en cambio":

- Presenta la evaluación global más completa de los recursos de agua dulce del planeta realizada hasta ahora. Y

- Pone de relieve el papel que este imprescindible líquido desempeña en el desarrollo y el crecimiento económico.

- El Informe, que se publica cada tres años, afirma:"Por la creciente escasez de agua, un gobierno adecuado es imprescindible para su gestión.....la lucha contra la pobreza depende también de nuestra capacidad para invertir en los recursos hídricos"

¿Es solución, la mejor gestión del agua? Para minimizar las pérdidas de agua, mejorar su gestión y reducir su demanda, existen ya políticas públicas. Muchos países han adoptado leyes para proteger sus recursos hídricos y administrarlos con prudencia. Sin embargo, estas medidas todavía no han surtido efectos palpables debido a que se limitan al sector del agua y se toman decisiones clave al margen del mismo.

\section{Precisiones I}

- En México, un país rico en recursos naturales se obtiene el agua que consume la población de diversas fuentes tales como ríos, arroyos y acuíferos del subsuelo.

- Estos acuíferos se recargan de forma natural en época de lluvias. Sin embargo, la duración de la época de lluvias es en promedio de cuatro meses lo que favorece una escasa captación. 
- Si del total de agua captada por lluvias, aprox. el $70 \%$ se evapora, la desproporción que existe entre la cantidad de agua que se capta por escurrimiento y las extensiones territoriales que comprenden hace que la disponibilidad del agua sea cada vez menor.

- México enfrenta actualmente graves problemas de disponibilidad, desperdicio y contaminación de agua.

\section{Precisiones II}

- Cabe recordar que existen diferencias territoriales importantes que son desfavorables:

- a)En el norte del territorio nacional, el agua de lluvia que se capta por escurrimiento es únicamente el $4 \%$ mientras que

- b) en el sureste y las zonas costeras se logra captar el 50\% del escurrimiento.

- En la zona sur del país, donde se localizan las regiones húmedas, las presas tienen como función almacenar el agua para la generación de la energía eléctrica y el control de avenidas.

- Por el contrario en la zona norte del país, al estar constituida por regiones áridas, las presas tienen la función de captar el agua que se utilizará en la actividad agrícola

\section{Precisiones III:}

En México, la gestión del agua se encuentra centralizada en la Comisión Nacional del Agua (CNA), a pesar del discurso oficial del proceso de descentralización de la gestión del agua, según el cual se ha pretendido

- materializar, entre otros instrumentos, en una amplísima reforma legislativa(2004) según la cual para 
- fortalecer la capacidad institucional de los gobiernos locales, y la de los usuarios, vía los consejos de cuenca.

\section{- Problemática}

"La falta de ejercicio de las facultades de comprobación de la Comisión Nacional del Agua (CNA) en la actualidad (y de sus homólogas en distintas épocas) del cumplimiento de las obligaciones a que se refiere la Ley de Aguas Nacionales y otros ordenamientos por parte de asignatarios y concesionarios de los derechos de Agua de la cuenca del Río Sonora, ha generado la sobreexplotación y agotamiento del acuífero de la costa de Hermosillo,"

\section{Más Precisiones técnicas IV}

¿Qué es una Cuenca? Es la unidad territorial delimitada por un parte aguas o divisoria, en donde ocurre el agua en distintas formas y esta se almacena o fluye hasta un punto de salida.

\section{¿Cuál es la función de una Cuenca;}

En la cuenca se llevan a cabo todos los procesos ecológicos, al coexistir los recursos agua, suelo, flora, fauna, otros recursos naturales relacionados con estos y el medio ambiente.

\section{Parte Segunda}

\section{La reforma legislativa de 2004 en México}

- Ofrece la posibilidad de que los gobiernos estatales asuman, por la vía de los convenios de coordinación, el ejercicio de determinadas funciones que corresponden a la esfera federal de atribuciones. 
- El gobierno del estado de Sonora a través de la Comisión Estatal del Agua, elaboró (2007) un proyecto de Convenio de Coordinación para la descentralización de la gestión del agua en la Cuenca del Río Sonora ${ }^{6}$

\section{Precisiones V}

- A cinco años de distancia del proyecto de Convenio de Descentralización de facultades en materia de gestión del vital líquido en la Cuenca del Río Sonora entre la Federación y el gobierno del Estado de Sonora, la situación poco o nada ha cambiado, puesto que so pretexto de la diferencia de colores partidistas en el ámbito federal (PAN) y el local(PRI) no se logró firmar el precitado Convenio ${ }^{7}$ .De allí que sea válido

- Confirmar la urgencia de dejar de lado las formas tradicionales de administración pública que habiendo llegado a su límite, enfrentan una tendencia mundial generalizada que expresa el cuestionamiento y reclamo de la sociedad y que obliga a cambiar hacia formas más incluyentes de gobernar. Ya que de otra suerte

\section{Precisiones VI}

Su implementación tendrá tal vez que:

- esperar a que lleguen los nuevos funcionarios locales que reemplazarán (Septiembre de 2015) a los hoy en turno y con suerte en las coordenadas políticas nacionales los funcionarios federales (2012) coincidan en las siglas partidistas y por lo mismo las voluntades políticas en la misma dirección.

\footnotetext{
${ }^{6}$ La cuenca del río sonora, la más grande de las 10 que se comprenden en el estado, cuenta con una superficie de 39,943 km2 y 14 municipios. Agua potable: 14 municipios omapas y 1 coapaes. Las ciudades más importantes dentro de la cuenca son: Hermosillo, Cananea y Ures.Los usos del agua en la cuenca del rio sonora y su volumen (millones de m3) son: agrícola 869.1, doméstico121; industrial 17.3 pecuario;12.0; turístico $\quad 0.2$; total1,019.6

${ }^{7}$ Resultado del Proyecto financiado con Fondos Mixtos Conacyt "Estudio de alternativas jurídico administrativas para proponer la administración conjunta del agua entre el Estado de Sonora y la federación", de tal forma que el Gobierno del estado asuma mayores atribuciones y responsabilidades relacionadas con el sector hidráulico en el territorio estatal, aplicando criterios de concurrencia subsidiariedad mediante la suscripción de un convenio en el marco del artículo 116 de la Constitución General de la República"
} 
O quizás esperar:

- a que la cuestión del agua se politice y

"las rivalidades emergentes" en relación al agua entre los gobiernos locales y el federal, entre diferentes sectores de actividad, y entre zonas rurales y urbanas" obstaculicen la seguridad local, la seguridad regional o la seguridad Binacional.

\section{A manera de propuestas que deriven un final más satisfactorio}

VIII. ¿Qué requieren hacer los gobiernos para que las medidas sean efectivas?

Participación es la clave e Imprescindible involucrar (participación) en ellas a responsables de muchos otros sectores como la agricultura, la energía, el comercio y la banca, porque ejercen una influencia decisiva en la gestión de los recursos hídricos

\section{Sin embargo estas decisiones requieren de:}

- 1) voluntad política que defina y traduzca en acciones concretas el discurso descentralizador del Estado.

- 2) constructiva discusión, con una agenda acordada entre gobiernos (local y federal) para

- a) definir los límites de la descentralización y

- b) convenir de forma clara las responsabilidades relacionadas con la gestión del agua y recursos presupuestales asignables, y la

- 3) participación de las empresas privadas y las organizaciones de la sociedad civil involucradas en el tema del agua.

A través de la participación se puede desencadenar círculos virtuosos: más participación puede llevar a políticas públicas más sensibles socialmente, y a inversiones sociales mayores. 
La participación es clave para que: la gente recupere su voz,las autoridades se responsabilicen de cada una de las funciones relacionadas con la gestión del agua,las sociedades sean más equilibradas y como bien señalara Don Antonio Fernández Poyato :

- la integración y convivencia en las ciudades propician la cohesión social y el desarrollo

¿Quién puede forzar que las autoridades (locales y federales), particulares y sociedad civil efectivamente se responsabilicen de cada una de las funciones relacionadas con la gestión del agua? ¿Cuál es el margen de participación óptimo que le corresponde a cada uno?

La respuesta se dará en la medida de la efectiva cooperación y coordinación entre los agentes involucrados, evidenciada en el beneficio individual y colectivo obtenido

En México uno de los factores que más contribuyen a debilitar el nivel municipal no es solo la centralización federal sino también y de manera más significativa la centralización a nivel de los estados.

Luego entonces se requiere promover la participación ciudadana en los nuevos espacios creados o modificados a fin de que en las discusiones y acuerdos de los órganos colegiados sean tomadas en cuenta e influyan en las decisiones de la autoridad y repercutan en una mejor calidad de vida. Asimismo reconocer y fortalecer en su caso las formas autogestoras de participación social que a lo largo de la historia de los pueblos han resuelto ejemplarmente problemas de abastecimiento y calidad del recurso hídrico. .

Aquí cabe recalcar que la propia iniciativa de los gobernantes presionados a su vez por las demandas ciudadanas es, la que generará, la viabilidad de diseñar mecanismos claros de consulta a los representados y de rendición de cuentas de los representantes, a fin de que la participación de las organizaciones sociales en los órganos de gobierno para la toma de decisiones refleje el verdadero sentir de los actores involucrados. 
Finalmente, es necesario señalar la importancia de que se reordene la actuación de la administración pública federal. Para ello se debe "transformar la práctica de elaborar programas de modernización de la administración pública federal de manera centralizada, en trabajo de "gabinetes" y sin contar con el consenso de los actores fundamentales.

La reforma administrativa pendiente debe contar con el apoyo y la colaboración de los actores involucrados, así como la voluntad política para llevarla a cabo"8. Además, se debe evitar duplicar esfuerzos y actuar en forma coordinada con los otros órdenes de gobierno en aras de resolver conjuntamente y en cohesión con la sociedad civil los grandes problemas nacionales.

\section{Referencias Bibliográficas}

Aboites Aguilar, Luis, (1998). El Agua de la Nación. Una historia política de México (1888-1946), Centro de Investigaciones y Estudios Superiores en Antropología Social, México.

Aboites Aguilar, Luis y Valeria Estrada Tena, (2004). Del agua municipal al agua nacional. Materiales para una historia de los municipios en México 1901-1945, CNA, Centro de Investigaciones y Estudios Superiores en Antropología Social, México.

Aboites Aguilar, Luis, (1997). Boletín del Archivo Histórico del Agua, año 4, número 11, septiembrediciembre.

Acosta Romero, Miguel, (2003). Segundo Curso de Derecho Administrativo, Segunda edición, Ed. Porrúa, México.

Cabrero, E. y R García, (1995).La nueva gestión municipal en México, Análisis de experiencias innovadoras en Gobiernos Locales. ED. Miguel Ángel Porrúa.

Denton Navarrete, Thalía, (2002) Tesis Doctoral el Régimen Jurídico del Agua en México.

González Beltrones Adrià Velia, (2007)"Las relaciones intergubernamentales y la participación ciudadana en la gestión integral del agua." pp.183-190 en Régimen jurídico del Agua .Culturas y Sistemas Jurídicos Comparados. Fernández Ruiz Jorge, Sánchez Javier Santiago (Coordinadores) UNAM ISBN 978-97032-4405-8

González Beltrones Adrià (2010). Agua: Asunto de seguridad nacional e importancia de la participación ciudadana en su gestión Cuestiones Jurídico Administrativas Locales, en Materia de Aguas del Siglo XX pp. 160, González Beltrones Adrià Velia y Aragón Salcido María Inés, UNISÓN.

Moreno Vásquez, José Luis, (2006). Por abajo del Agua, Sobreexplotación y agotamiento del Acuífero de la Costa de Hermosillo, 1945-2005, Colegio de Sonora.

Sánchez González José Juan, (2004). Reforma, modernización e innovación en la historia de la Administración Pública en México. ED. IAPQRO.

Tena Ramírez, Felipe. (2002). Derecho Constitucional Mexicano, edición XXXI, Ed. Porrúa, México.

\footnotetext{
${ }^{8}$ Sánchez González José Juan Reforma, modernización e innovación en la historia de la Administración Pública en México .pp 78 ED. IAPQRO, 2004.
} 